[15] M. Neumann, Continuity of sublinear operators of F-spaces, Manuscripta Math. 26 (1978), 37-61.

[16] -, Automatic continuity of linear operators, in: Functional Analysis: Surveys and Recent Results II, North-Holland Math. Studies 38, 1980, 269-296.

[17] -, Uniform boundedness and closed graph theorems for convex operators, Math. Nachr. (to appear).

[18] J. Peetre, Rectification à larticle "Une caractérisation abstraite des opérateurs différentiels", Math. Scand. 8 (1960), 116-120.

[19] A. L. Peressini, Ordered Topological Vector Spaces, Harper and Row, New York 1967.

[20] V. Pták, A uniform boundedness theorem and mappings into spaces of operators, Studia Math. 31 (1968), 425-431.

[21] A. M. Sinclair, Automatic continuity of linear operators, London Math. Soc. Lect. Notes Series 21, Cambridge University Press, Cambridge 1976.

[22] J. D. Stein Some aspects of automatic continuity, Pacific J. Math. 50 (1974), 187-204.

[23] Y.-C. Wong and K.-F. Ng, Partially Ordered Topological Vector Spaces, Clarendon Press, Oxford 1973.

FACHBEREICH MATHEMATIK, UNIVERSITAT ESSEN-GHS

D-4300 Essen, West Germany

and

INSTITUTE OF MATHEMATICS,

CZECHOSLOVAK ACADEMY OF SCIENCES

Žtiná 25, 11567 Prahn 1, Crechoslovakis

\section{Some combinatorial and probabilistic inequalities and their application to Banach space theory}

by

STANISŁAW KWAPIEŃ (Warszawa) and CARSTEN SCHUTTT (Kiel)

Abstract. Some combinatorial and probabilistic estimates are proved. As applications they are used to study invariants of Banach spaces, such as the projection constant.

Introduction. We consider here for $x, y \in \boldsymbol{R}^{n}, 1 \leqslant p \leqslant \infty$,

$$
\underset{\pi}{\operatorname{Ave}}\left\|\left(x_{i} y_{\pi(i)}\right)_{i=1}^{n}\right\|_{p}
$$

and give the order of this expression in terms of the vectors $x$ and $y$. For special vectors $x$ or $y$ this was already considered by. E. D. Gluskin [4] and, independently, in [7].

It seems that the estimates that we obtain are, in a sense, crucial if one wants to compute projection constants of symmetric Banach spaces and related invariants.

We give some examples and applications. We characterize the symmetric sublattices of $l^{1}\left(c_{0}\right)$ and the symmetric subspaces of $l^{1}$. We compute the positive projection constant of a (nnite-dimensional) Orlicz space and show that it is, up to a universal constant, the same as the one of the dual space. For symmetric spaces this is in general not true.

The order of the projection constant of the Lorentz space $l_{n}^{2,1}, n \in N$, is estimated. The result seems to be rather peculiar.

We are grateful to J. Lindenstrauss and G. Schechtman for discussions.

0. Preliminaries. In this paper we are mainly concerned with finitedimensional Banach spaces that have a 1-symmetric basis, i.e. a basis $\left\{e_{i}\right\}_{i=1}^{n}$ such that for all $a_{i} \in \boldsymbol{R}, \varepsilon_{i}= \pm 1, i=1, \ldots, n$ and all permutations $\pi$ we have

$$
\left\|\sum_{i=1}^{n} a_{i} e_{i}\right\|=\left\|\sum_{i=1}^{n} \varepsilon_{i} a_{i} e_{n(i)}\right\| .
$$

The projection constant of a finite-dimensional Banach space $E$ is given by $\gamma_{\infty}(E)=\inf \left\{\|P\| \mid P\right.$ is a projection from $l^{\infty}$ onto $\left.E\right\}$. 
And for spaces with 1-symmetric bases we can define the positive projection constant

$$
\gamma_{\infty}^{+}(E)=\operatorname{supinf}_{\boldsymbol{F} \supseteq \boldsymbol{E}}\{\|P\| \mid P \text { is a positive projection from } F \text { onto } E\}
$$

where the supremum is taken over all lattices $F$ that contain $E$ as a sublattice.

The notions from Banach space theory we use are standard and can be found in [5].

1. Combinatorial estimates. We consider here the set $\mathcal{S}$ of all permutations of $\{1,2, \ldots, n\}$. On this set we introduce the measure $\mu$ with $\mu(\pi)=1 / n$ ! for each $\pi \in \subseteq$ . Thus the integral equals the average over all permutations:

$$
\int_{\Xi} f(\pi) d \mu(\pi)=\frac{1}{n !} \sum_{\pi \in \Xi} f(\pi) .
$$

Suppose that $a=\left(a_{i, j}\right)_{1 \leqslant i, j \leqslant n} \in \boldsymbol{R}^{n \times n}$ is a matrix with $n$ rows and $n$ columns. Then $s(k), k=1,2, \ldots, n^{2}$ denotes the nonincreasing rearrangement of $\left\{\left|a_{i, j}\right|: 1 \leqslant i, j \leqslant n\right\}$. This notation will be used throughout this section.

THEOREM 1.1. For all $n \in N$ and all $a \in R^{n \times n}$ we have

$$
\frac{1}{2} n^{-1} \sum_{k=1}^{n} s(k) \leqslant \int_{\Xi} \max _{1 \leqslant i \leqslant n}\left|a_{i, \pi(i)}\right| d \mu(\pi) \leqslant n^{-1} \sum_{k=1}^{n} s(k) .
$$

Proof. Let us observe that both expressions in the inequality define norms on $\boldsymbol{R}^{n \times n}$. Therefore to prove the right-hand inequality it is enough to check it for the extreme points in the unit ball of the norm given by the expression on the right side of the inequality. However, it is easy to see that these extreme points are matrices $a$ such that $\left|a_{i, j}\right|=1$ for at least $n$ pairs of $(i, j)$ and $a_{i, j}=0$ for all other $(i, j)$, or such that $\left|a_{i_{0}, j_{0}}\right|=n$ for a pair $\left(i_{0}, j_{0}\right)$ and $a_{i, j}=0$ for all other $(i, j)$. For such matrices it is very easy to verify the inequality.

To prove the left-hand inequality it is enough to consider only matrices $a$ with at most $n$ terms different from 0 . This is so, because if we replace by 0 numbers which do not enter into $s(k), k=1,2, \ldots, n$, then the left side of the inequality will not change, while the right side will not increase. We may also assume that there are no two different from 0 terms in the same row or column. This is so, because if we move any term $a_{j, k}$ into row $l$ and column $m$ so that there are no other terms different from 0 in row $l$ and in column $m$ and if we put 0 instead of $a_{j, k}$, then for the new matrix the left side will be the same as for the old one, while the right side will not increase. To see this let us denote by $a^{\prime}$ the new matrix and let $*: \mathfrak{S} \rightarrow \mathfrak{S}$ be the map defined as follows: if $\pi(j)=k$ and $\pi(l) \neq m$ then $\pi^{*}(l)=m, \pi^{*}\left(\pi^{-1}(m)\right)=k, \pi^{*}(j)=\pi(l)$, and $\pi^{*}(t)=\pi(t)$ for all other $t$;

if $\pi(j) \neq k$ and $\pi(l)=m$ then $\pi^{*}(j)=k, \pi^{*}\left(\pi^{-1}(k)\right)=m, \pi^{*}(l)=\pi(j)$, and $\pi^{*}(t)=\pi(t)$ for all other $t$

for all other $\pi$ let $\pi^{*}=\pi$. Then it is easy to see that for all $\pi \in \subseteq$ we have $\pi^{* *}=\pi$ and

$$
\max _{1 \leqslant i \leqslant n}\left|a_{i, \pi(i)}^{\prime}\right|+\max _{1 \leqslant i \leqslant n}\left|a_{i, \pi^{*}(i)}^{\prime}\right| \leqslant \max _{1 \leqslant i \leqslant n}\left|a_{i, \pi(i)}\right|+\max _{1 \leqslant i \leqslant n}\left|a_{i, \pi^{*}(i)}\right| .
$$

This justifies our claim.

Thus, without loss of generality we may assume that all numbers in the matrix which are different from 0 are placed on the diagonal of the matrix. Therefore, taking the average, we will obtain a new matrix with constant terms on the diagonal and such that the right side will not increase, while the left side will remain unchanged. So, the whole proof is reduced to verifying the inequality for the diagonal matrix with all terms on the diagonal equal to 1. For this matrix the right side of the inequality is equal to

$$
1-\frac{1}{2 !}+\frac{1}{3 !}-\ldots+(-1)^{n+1} \frac{1}{n !} \geqslant \frac{1}{2}
$$

cf. [2], Ch. $4, \S 1$.

THeorem 1.2. For $1 \leqslant p \leqslant \infty$ and all $n \in N$ and $a \in \boldsymbol{R}^{n \times n}$ we have

$$
\begin{aligned}
& \frac{1}{5}\left\{n^{-1} \sum_{k=1}^{n} s(k)+\left(n^{-1} \sum_{k=n+1}^{n^{2}} s(k)^{p}\right)^{1 / p}\right\} \\
& \quad \leqslant \int_{\odot}\left(\sum_{i=1}^{n}\left|a_{i, \pi(i)}\right|^{p}\right)^{1 / p} d \mu(\pi) \leqslant n^{-1} \sum_{k=1}^{n} s(k)+\left(n^{-1} \sum_{k=n+1}^{n^{2}} s(k)^{p}\right)^{1 / p} .
\end{aligned}
$$

Proof. Let $a^{\prime \prime}$ be the matrix defined by $a_{i, j}^{\prime \prime}=a_{i, j}$ if $a_{i, j}$ in the nonincreasing rearrangement into $s(k), k=1,2, \ldots, n^{2}$ has the index less than or equal to $n$, and let $a_{i, J}^{\prime \prime}=0$ in the opposite case. Then for the matrix $a^{\prime}=a-a^{\prime \prime}$ we have $\left|a_{i, j}^{\prime}\right| \leqslant s(n)$ for $1 \leqslant i, j \leqslant n$. By the triangle inequality we obtain

$$
\begin{aligned}
\int_{\Theta}\left(\sum_{i=1}^{n}\left|a_{i, \pi(i)}\right|^{1 / p} d \mu(\pi)\right. & \leqslant \int_{\Theta}\left(\sum_{i=1}^{n}\left|a_{i, \pi(i)}^{\prime}\right|^{p}\right)^{1 / p} d \mu(\pi)+\int_{\Xi}\left(\sum_{i=1}^{n} \mid a_{i, \pi(i)}^{\prime \prime}\right)^{p / p} d \mu(\pi) \\
& \left.\leqslant\left(\int_{\Theta}\left(\sum_{i=1}^{n} \mid a_{i, \pi(i)}^{\prime}\right)^{p}\right) d \mu(\pi)\right)^{1 / p}+\int_{\Theta}\left(\sum_{i=1}^{n}\left|a_{i, \pi(i)}^{\prime \prime}\right|\right) d \mu(\pi) \\
& =\left(n^{-1} \sum_{k=n+1}^{n^{2}} s(k)^{p}\right)^{1 / p}+n^{-1} \sum_{k=1}^{n} s(k) .
\end{aligned}
$$


To prove the left-hand inequality let us denote

$$
f(\pi)=\left(\sum_{i=1}^{n} \mid a_{i, \pi(i)}^{\prime}\right)^{1 / p}
$$

for simplicity. By the Hölder inequality we obtain

$$
\int_{\Xi} f^{p} d \mu \leqslant\left(\int_{\Xi} f d \mu\right)^{p /(2 p-1)}\left(\int_{\Xi} f^{2 p} d \mu\right)^{(p-1) /(2 p-1)} .
$$

By simple computations we obtain

$$
\begin{aligned}
\int_{\Xi} f^{2 p}(\pi) d \mu(\pi) & =\frac{1}{n(n-1)} \sum_{\substack{i \neq k \\
j \neq l}}\left|a_{i, j}^{\prime}\right|^{p}\left|a_{k, l}^{\prime}\right|^{p}+\frac{1}{n} \sum_{1 \leqslant i, j \leqslant n}\left|a_{i, j}^{\prime}\right|^{2 p} \\
& \leqslant \frac{n}{n-1}\left(\int_{\Xi} f^{p}(\pi) d \mu(\pi)\right)^{2}+s(n)^{p} \int_{\Xi} f^{p}(\pi) d \mu(\pi) .
\end{aligned}
$$

By Theorem 1.1 it follows that

$$
s(n) \leqslant n^{-1} \sum_{k=1}^{n} s(k) \leqslant 2 \int_{\subseteq} \max _{1 \leqslant i \leqslant n}\left|a_{i, \pi(i)}\right| d \mu(\pi) \leqslant 2 \int_{\odot}\left(\sum_{i=1}^{n}\left|a_{i, \pi(i)}\right|^{p}\right)^{1 / p} d \mu(\pi) .
$$

Let

$$
y=\left\{\int_{\Xi}\left(\sum_{i=1}^{n}\left|a_{i \pi(i)}\right|^{p}\right)^{1 / p} d \mu(\pi)\right\}^{p} \quad \text { and } \quad x=\int_{\Xi} f^{p}(\pi) d \mu(\pi)=n^{-1} \sum_{k=n+1}^{n^{2}} s(k)^{p} .
$$

Combining the above inequalities, we get

$$
x^{2 p-1} \leqslant y\left(2 x^{2}+2^{p} y x\right)^{p-1}
$$

or, which is the same,

$$
\varphi\left(\frac{x}{y}\right)=\left(\frac{x}{y}\right)^{p /(p-1)}-2 \frac{x}{y}-2^{p} \leqslant 0 .
$$

Since the function $\varphi(\lambda)$ is increasing for $\lambda>3^{p}$ and $\varphi\left(3^{p}\right)>0$, we conclude that $x / y<3^{p}$. This together with Theorem 1.1 proves that

$$
n^{-1} \sum_{k=1}^{n} s(k)+\left(n^{-1} \sum_{k=n+1}^{n^{2}} s(k)^{p}\right)^{1 / p} \leqslant 5 \int_{\Theta}\left(\sum_{i=1}^{n}\left|a_{i, \pi(i)}\right|^{p}\right)^{1 / p} d \mu(\pi) .
$$

2. Symmetric sublattices of $l^{1}\left(c_{0}\right)$ and symmetric subspaces of $l^{1}$. If $M$ is an - Orlicz function, then $M^{*}$ denotes its complementary function [5]. Let $y \in \boldsymbol{R}^{n}$ be such that $y_{1} \geqslant y_{2} \geqslant \ldots \geqslant y_{n}>0$ with $\sum_{i=1}^{n} y_{i}=1$. Then we can associate with $y$ a convex function $M$ with $M(0)=0$ and $M(1)=1$ by putting

$$
M\left(\sum_{i=1}^{k} y_{i}\right)=k / n \quad \text { for } k=1, \ldots, n
$$

and extending it (e.g. linearly).

Lemma 2.1. Let $y \in \boldsymbol{R}^{n}$ with $y_{1} \geqslant y_{2} \geqslant \ldots \geqslant y_{n}>0$ and $\sum_{i=1}^{n} y_{i}=1$ and let $M$ be a convex function satisfying (2.1). Moreover, let

$$
\begin{gathered}
B_{M}=\left\{x \in \boldsymbol{R}^{n} \mid \sum_{i=1}^{n} M\left(\left|x_{i}\right|\right) \leqslant 1\right\}, \\
B=\text { convex hull }\left\{\left(\varepsilon_{i} \sum_{j=1}^{l_{i}} y_{j}\right)_{i=1}^{n} \mid \sum_{i=1}^{n} l_{i}=n, \varepsilon_{i}= \pm 1, i=1, \ldots, n\right\} .
\end{gathered}
$$

Then we have

$$
B \subseteq B_{M} \subseteq 3 B \text {. }
$$

Proof. To prove the left-hand inclusion we just have to observe that

$$
\sum_{i=1}^{n} M\left(\left|\varepsilon_{i} \sum_{j=1}^{l_{i}} y_{j}\right|\right)=\sum_{i=1}^{n} l_{i} / n=1 .
$$

On the other hand, let

$$
\sum_{i=1}^{n} M\left(x_{i}\right)=1 \quad \text { with } x_{1} \geqslant x_{2} \geqslant \ldots \geqslant x_{n} \geqslant 0
$$

and let

$$
x=\tilde{x}+\hat{x}=\left(x_{1}, \ldots, x_{r}, 0, \ldots, 0\right)+\left(0, \ldots, 0, x_{r+1}, \ldots, x_{n}\right)
$$

be such that $M\left(x_{i}\right)>1 / n$ for $1 \leqslant i \leqslant r$ and $M\left(x_{i}\right) \leqslant 1 / n$ for $r<i \leqslant n$. Since $M\left(y_{1}\right)=1 / n$, we have $\hat{x} \leqslant\left(y_{1}, \ldots, y_{1}\right)$. Moreover, since $\left(y_{1}, \ldots, y_{1}\right) \in B$, we get $\hat{x} \in B$.

On the other hand, for every $i, 1 \leqslant i \leqslant r$, there is a $k_{i}$ with

$$
k_{i} / n<M\left(x_{i}\right) \leqslant\left(k_{i}+1\right) / n \text {. }
$$

Since

$$
\sum_{i=1}^{r} k_{i} / n \leqslant \sum_{i=1}^{r} M\left(\sum_{j=1}^{k_{i}} y_{j}\right) \leqslant \sum_{i=1}^{r} M\left(x_{i}\right) \leqslant 1
$$

we conclude that

$$
z=\left(\sum_{j=1}^{k_{1}} y_{j}, \ldots, \sum_{j=1}^{k_{r}} y_{j}, 0, \ldots, 0\right) \in B
$$


Moreover, we have by (2.2)

$2 z=\left(2 \sum_{j=1}^{k_{1}} y_{j}, \ldots, 2 \sum_{j=1}^{k_{r}} y_{j}, 0, \ldots, 0\right) \geqslant\left(\sum_{j=1}^{k_{1}+1} y_{j}, \ldots, \sum_{j=1}^{k_{r}+1} y_{j}, 0, \ldots, 0\right) \geqslant \tilde{x}$.

Thus $\tilde{x} \in 2 B$ and $\hat{x}+\tilde{x} \in 3 B$.

Corollary 2.2. There is a $C>0$ such that for all $n \in N, y \in \boldsymbol{R}^{n}, y_{1} \geqslant y_{2}$ $\geqslant \ldots \geqslant y_{n}>0$ and $\sum_{i=1}^{n} y_{i}=1$ we have

$$
\frac{1}{C n}\|x\|_{*} \leqslant \int_{\Xi} \max _{1 \leqslant i \leqslant n}\left|x_{i} y_{\pi(i)}\right| d \mu(\pi) \leqslant \frac{C}{n}\|x\|_{*}
$$

where \|\|$_{*}$ denotes the norm of $\left(l_{n}^{M}\right)^{*}$ with $M$ satisfying (2.1),

On the other hand, for every Orlicz function $M$ there is a $y \in \boldsymbol{R}^{\prime \prime}$ such that the above estimates are satisfied.

This corollary is proved by Theorem 1.1 and Lemma 2.1 .

We say that a norm is an average of Orlicz norms if

$$
\|x\|=\sum_{i=1}^{\infty}\left|a_{i}\right|\|x\|_{M_{i}}
$$

with $\sum\left|a_{i}\right|=1$. We call 'such a space an average of Orlicz spaces.

THEOREM 2.3. There is a $C>0$ with the following property: $E$ is a finitedimensional symmetric sublattice of $l^{1}\left(c_{0}\right)$ if and only if $E$ is $C$-order isomorphic to an average of Urlicz spaces.

Proof. Let $\left\{y_{i}\right\}_{i=1}^{n}$ be a 1 -symmetric, normalized basis of a sublattice. Then, because of symmetricity, we get

$$
\left\|\sum_{i=1}^{n} a_{i} y_{i}\right\|=\sum_{k=1}^{\infty} \max _{1 \leqslant l \leqslant \infty}\left|\sum_{i=1}^{n} a_{i} y_{i}(k, l)\right|=\sum_{k=1}^{\infty} \text { Ave } \max _{1 \leqslant l \leqslant \infty}\left|\sum_{i=1}^{n} a_{\pi(i)} y_{i}(k, l)\right| .
$$

Since the vectors $y_{i}$ are disjoint, we have

$$
\left\|\sum_{i=1}^{n} a_{i} y_{i}\right\|=\sum_{k=1}^{\infty} \underset{\pi}{\text { Ave }} \max _{1 \leqslant l \leqslant \infty} \max _{1 \leqslant i \leqslant n}\left|a_{\pi(i)} y_{i}(k, l)\right| .
$$

Now we put $z_{i}(k):=\max _{l} y_{i}(k, l)$ and obtain

$$
\left\|\sum_{i=1}^{n} a_{i} y_{i}\right\|=\sum_{k=1}^{\infty} \underset{\pi}{\text { Ave }} \max _{1 \leqslant i \leqslant n}\left|a_{\pi(i)} z_{i}(k)\right| .
$$

Now we apply Corollary 2.2. In order to find all averages of Orlicz spaces as $C$-order isomorphic copies of sublattices of $l^{1}\left(c_{0}\right)$ we apply again Corollary 2.2.
COROLlary 2.4. There is a constant $C>0$ such that for all $n \in N$ and all $x \in \boldsymbol{R}^{n}$ we have

$$
C^{-1}\|x\|_{2} \leqslant \sqrt{n} \int_{\Xi} \max _{1 \leqslant i \leqslant n} \frac{\left|x_{i}\right|}{\sqrt{\pi(i)}} d \mu(\pi) \leqslant C\|x\|_{2} .
$$

Proof. We see immediately that for $y=(1 / \sqrt{n i})_{i=1}^{n}$ we get in (2.1) $M^{-1}(k / n) \sim \sqrt{k / n}$. Thus the result follows from Corollary 2.2.

Lemma 2.5 [7]. Let $\left\{e_{i}\right\}_{i=1}^{n}$ be a 1-symmetric basis of $E$. Then

$$
\gamma_{\infty}^{+}(E)=\inf \max _{\left\|x^{*}\right\|=1} \sum_{l=1}^{N} \max _{l \leqslant l \leqslant n}\left|\left\langle x^{*}, e_{i}\right\rangle\left\langle x_{l}, e_{i}^{*}\right\rangle\right|
$$

where the infimum is taken over all sequences satisfying $\sum_{l=1}^{N}\left\|x_{l}\right\|=n$.

With this lemma and Corollary 2.4 we obtain the following corollary. This has been done in ([7], Prop. 3.2).

Corollary 2.6. Let $\left\{e_{i}\right\}_{i=1}^{n}$ be a 1-symmetric basis of $E$ and id $\in L\left(l_{n}^{2}, E\right)$ the natural identity $\mathrm{id}\left(\left(a_{i}\right)_{i=1}^{n}\right)=\sum_{i=1}^{n} a_{i} e_{i}$. Then we have

$$
\sqrt{n}\left(\|\mathrm{id}\|\left\|\sum_{k=1}^{n} \frac{1}{\sqrt{k}} e_{k}^{*}\right\|\right)^{-1} \leqslant C \gamma_{\infty}^{+}(E)
$$

where $C$ is a constant not depending on $n$.

Corollary 2.7. There is a $C>0$ such, that for all $n \in N$ we have

(i) $C^{-1} \sqrt{n / \ln n} \leqslant \gamma_{\infty}^{+}\left(l_{n}^{2, r}\right) \leqslant C \sqrt{n / \ln n} \quad$ if $1 \leqslant r \leqslant 2$,

(ii) $C^{-1} \sqrt{n}(\ln n)^{-1 / r^{*}} \leqslant \gamma_{\infty}^{+}\left(l_{n}^{2, r}\right) \leqslant C \sqrt{n}(\ln n)^{-1 / r^{*}}$ if $2 \leqslant r \leqslant \infty$.

Proof. The left-hand inequalities are consequences of Corollary 2.6. The right-hand inequality (i) follows from Corollary 2.4 and Lemma 2.5 if one chooses as a sequence

$$
\frac{n}{n !}\left(\sum_{k=1}^{n} 1 / k\right)^{-1 / 2}(1 / \sqrt{\pi(i)})_{i=1}^{n}
$$

with $\pi$ a permutation. For the right-hand inequality in (ii) we choose instead the sequence used in ([7], Proof of Theorem 3.1) to get the estimate for $l_{n}^{2}$.

THEOREM 2.8 (cf. also Corollary 4.3). There is a constant $C>0$ such that for all Orlicz functions we have

$$
C^{-1} \gamma_{\infty}^{+}\left(l_{n}^{M}\right) \leqslant \gamma_{\infty}^{+}\left(l_{n}^{M^{*}}\right) \leqslant C \gamma_{\infty}^{+}\left(l_{n}^{M}\right) .
$$

Proof. By Theorem 2.3, $l_{n}^{M}$ is $C$-isomorphic to a sublattice $E$ of $l^{1}\left(l_{n}^{\infty}\right)$. 
Thus there is a positive projection from $l^{1}\left(l_{n}^{\infty}\right)$ onto $E$ with $\|P\| \leqslant \gamma_{\infty}^{+}\left(l_{n}^{M}\right)$. By dualization it follows that the identity on $E^{*}$ can be positively factored with the same bound. By Lemma 2.1 of [7] it follows that

$$
\gamma_{\infty}^{+}\left(l_{n}^{M^{4}}\right) \leqslant C^{\prime} \gamma_{\infty}^{+}\left(l_{n}^{M}\right)
$$

It was pointed out to us by Gideon Schechtman, Rehovot, that the estimate of Theorem 1.2 for $p=2$ gives a characterization of the finite-dimensional symmetric subspaces of $l^{1}$. The infinite-dimensional version of this characterization was proved by Dacunha-Castelle [1]. It seems that the arguments in [1] were purely infinite-dimensional and that the finite-dimensional version could not be obtained from them.

The following arguments are given here with the permission of Gideon Schechtman. Let

$$
M(t)= \begin{cases}(2-1 / n)^{-1} n t^{2} & \text { if } t \leqslant 1 / n \\ (2-1 / n)^{-1}(2 t-1 / n) & \text { if } t>1 / n\end{cases}
$$

$M$ is an Orlicz function.

LeMmA 2.9. Let $a_{1} \geqslant a_{2} \geqslant \ldots \geqslant a_{n^{2}} \geqslant 0$. Then we have

$$
(4 n)^{-1}\left\|\left(a_{i}\right)_{i=1}^{n^{2}}\right\|_{M} \leqslant n^{-1} \sum_{i=1}^{n} a_{i}+\left(n^{-1} \sum_{i=n+1}^{n^{2}} a_{i}^{2}\right)^{1 / 2} \leqslant 6 n^{-1}\left\|\left(a_{i}\right)_{i=1}^{n_{2}}\right\|_{M} .
$$

Proof. Assume that $\|a\|_{M} \leqslant 1 / 2$. Since $2 M(t) \leqslant M(2 t)$, we have

$$
(2-1 / n)^{-1}\left\{\sum_{a_{i}>1 / n}\left(2 a_{i}-1 / n\right)+n \sum_{a_{i} \leqslant 1 / n} a_{i}^{2}\right\} \leqslant 1 / 2 .
$$

In particular, we get

$$
\sum_{a_{i}>1 / n} a_{i} \leqslant \sum_{a_{i}>1 / n}\left(2 a_{i}-1 / n\right) \leqslant 1 .
$$

Therefore we have

$$
\sum_{i=1}^{n} a_{i} \leqslant 2 \quad \text { and } \quad \operatorname{card}\left\{i \mid a_{i}>1 / n\right\} \leqslant n .
$$

It follows that

$$
\sum_{i=1}^{n} a_{i}+\left(n^{-1} \sum_{i=n+1}^{n^{2}} a_{i}^{2}\right)^{1 / 2} \leqslant 3
$$

To prove the left-hand inequality assume

$$
\sum_{i=1}^{n} a_{i}+n\left(n^{-1} \sum_{i=n+1}^{n^{2}} a_{i}^{2}\right)^{1 / 2} \leqslant 1
$$

Clearly, we have

$$
n \sum_{a_{i} \leqslant 1 / n} a_{i}^{2} \leqslant n\left(\sum_{i=n+1}^{n^{2}} a_{i}^{2}+\sum_{\substack{a_{i} \leqslant 1 / n \\ i \leqslant n}} a_{i}^{2}\right) \leqslant n(1 / n+1 / n)=2,
$$

and, moreover, since $a_{n+1} \leqslant a_{n} \leqslant n^{-1} \sum_{i=1}^{n} a_{i} \leqslant 1 / n$, we have

$$
\sum_{a_{i}>1 / n} a_{i} \leqslant \sum_{i=1}^{n} a_{i} \leqslant 1
$$

Therefore we get

$$
\sum_{i=1}^{n^{2}} M\left(a_{i}\right)=(2-1 / n)^{-1}\left\{\sum_{a_{i}>1 / n}\left(2 a_{i}-1 / n\right)+n \sum_{a_{i} \leqslant 1 / n} a_{i}^{2}\right\} \leqslant 4 .
$$

Let $y \in \boldsymbol{R}^{n}, y \geqslant 0$, and

$$
M_{y}(t)=\sum_{i=1}^{n} M\left(t y_{i}\right) / \sum_{i=1}^{n} M\left(y_{i}\right)
$$

where $M$ is defined by (2.3). Note that $M_{y}$ is an Orlicz function.

Lemma 2.10. There is a $C>0$ such that for all $n \in \boldsymbol{N}$ and all $x, y \in \boldsymbol{R}^{n}$, $y \geqslant 0$, we have

$$
C^{-1}\|x\|_{M_{y}} \leqslant n\left(\sum_{i=1}^{n} M\left(y_{i}\right)\right)^{-1} \underset{\pi}{\operatorname{Ave}}\left(\sum_{i=1}^{n}\left|x_{i} y_{\pi(i)}\right|^{2}\right)^{1 / 2} \leqslant C\|x\|_{M_{y}} .
$$

Lemma 2.10 follows immediately from Theorem 1.2 and Lemma 2.9.

THeorem 2.11. There is a $C>0$ such that every finite-dimensional symmetric subspace of $l^{1}$ is $C$-isomorphic to an average of Orlicz spaces.

Proof. Let $\left\{y_{i}\right\}_{i=1}^{n}$ be a 1-symmetric basis of a subspace of $l^{1}$. By symmetricity and the Khintchine inequality it follows that

$$
\left\|\sum_{i=1}^{n} a_{i} y_{i}\right\|
$$

is up to a factor $\sqrt{2}$ the same as

$$
\sum_{k=1}^{\infty} \underset{n}{\operatorname{Ave}}\left(\sum_{i=1}^{n}\left|a_{n(i)} y_{i}(k)\right|^{2}\right)^{1 / 2} .
$$

Now we have to apply Lemma 2.10 .

3. The projection constant of $l_{n}^{2,1}$.

LEMMA 3.1. Let $\left\{e_{i}\right\}_{i=1}^{n}$ be a 1-symmetric basis of the Banach space $E$. 
Then we have

$$
\gamma_{\infty}(E) \leqslant \inf \max _{\left\|x^{*}\right\|=1} \sum_{l=1}^{N} \frac{1}{n !} \sum_{\pi}\left(\sum_{i=1}^{n}\left|\left\langle x^{*}, e_{\pi(i)}\right\rangle\left\langle x_{l}, e_{i}^{*}\right\rangle\right|^{2}\right)^{1 / 2} \leqslant \sqrt{2} \gamma_{\infty}(E)
$$

where the infimum is taken over all finite sequences with $\sum_{l=1}^{N}\left\|x_{l}\right\|=n$.

This lemma easily follows from the equality $n=\gamma_{\infty}(E) \pi_{1}(E)$, the Khintchine inequality and the 1-symmetricity of the basis $\left\{e_{i}\right\}_{i=1}^{n}$.

THEOREM 3.2. There are constants $C_{1}, C_{2}>0$ such that for all $n \in N$, $n \geqslant 5$, we have

$$
C_{1} \sqrt{\frac{n}{\ln \ln n}} \leqslant \gamma_{\infty}\left(l_{n}^{2,1}\right) \leqslant C_{2} \sqrt{\frac{n}{\ln \ln n}} .
$$

Proof. By Lemma 3.1 and since it is enough to consider only extreme points $x^{*}=(1 / \sqrt{k})_{k=1}^{n}-$ up to signs and permutations - of the dual unit ball we obtain for the projection constant

or by Theorem 1.2,

$$
\inf \sum_{l=1}^{N} \frac{1}{n !} \sum_{n}\left(\sum_{i=1}^{n}\left|\frac{x_{l}(i)}{\sqrt{\pi(i)}}\right|^{2}\right)^{1 / 2}
$$

$$
\inf \sum_{l=1}^{N}\left\{n^{-1} \sum_{k=1}^{n} s_{l}(k)+\frac{1}{\sqrt{n}}\left(\sum_{k=n+1}^{n^{2}}\left|s_{l}(k)\right|^{2}\right)^{1 / 2}\right\}
$$

where $s_{l}$ denote the decreasing rearrangements. By Corollary 2.4 we know that

$$
n^{-1} \sum_{k=1}^{n} s_{l}(k)
$$

is up to a constant equal to $n^{-1 / 2}\left\|x_{i}\right\|_{2}$. Thus we obtain for the projection constant

$$
\inf \sum_{l=1}^{N} \frac{1}{\sqrt{n}}\left\{\left\|x_{l}\right\|_{2}+\left(\sum_{k=n+1}^{n^{2}}\left|s_{l}(k)\right|^{2}\right)^{1 / 2}\right\}
$$

In order to estimate this expression from above we just consider the vector $x=(1 /(\sqrt{i} \ln 2 i))_{i=1}^{n}$ with $\|x\|_{2,1}=\sum_{i=1}^{n} 1 /(i \ln 2 i) \leqslant 2 \ln \ln n, n \geqslant 5$. Let $C>0$ be such that $\sum_{j=1}^{n} 1 /\left(j \ln ^{2} 2 j\right) \leqslant C$. Then the set

$$
\begin{aligned}
\left\{\frac{1}{\sqrt{i j} \ln 2 j} \mid j=1, \ldots, n, i=\right. & \left.1, \ldots,\left[n /\left(C j \ln ^{2} 2 j\right)\right]\right\} \\
& =\left\{\frac{1}{\sqrt{i j} \ln 2 j} \mid C i j \ln ^{2} 2 j \leqslant n, 1 \leqslant i, j \leqslant n\right\}
\end{aligned}
$$

is contained in the set of the greatest $n$ numbers of

$$
\left\{\frac{1}{\sqrt{i j} \ln 2 j} \mid 1 \leqslant i, j \leqslant n\right\} \text {. }
$$

Indeed, the cardinality is less than

$$
\sum_{j=1}^{n} \frac{n}{C j \ln ^{2} 2 j} \leqslant n
$$

Now, (3.1) is - up to a constant - less than

$$
\begin{aligned}
\frac{n}{\ln \ln n} \cdot \frac{1}{\sqrt{n}} & \left\{\left(\sum_{i=1}^{n} \frac{1}{i \ln ^{2} 2 i}\right)^{1 / 2}+\left(\sum_{j=1}^{n} \sum_{i=[n /(C j \ln 2}^{n} 2 j\right)\right] \\
& \leqslant C^{\prime} \frac{\sqrt{n}}{\ln \ln n}\left\{1+\left(\sum_{j=1}^{n} \frac{\ln \left(C j \ln ^{2} 2 j\right.}{\left.\ln ^{2} j\right)}\right)^{1 / 2}\right\} \\
& \leqslant C^{\prime \prime} \sqrt{\frac{n}{\ln \ln n}} .
\end{aligned}
$$

In order to estimate (3.1) from below we recall that for $x \in \boldsymbol{R}^{n}, x_{1} \geqslant x_{2} \geqslant \ldots$ $\ldots \geqslant x_{n} \geqslant 0$ there are natural numbers $l_{1} \geqslant l_{2} \geqslant \ldots \geqslant l_{n} \geqslant 0$ such that $\sum_{i=1}^{n} l_{i}=n$ and $\left\{x_{i} / \sqrt{j} \mid 1 \leqslant j \leqslant l_{i}, 1 \leqslant i \leqslant n\right\}$ are the greatest $n$ numbers of $\left\{x_{i} / \sqrt{j} \mid 1 \leqslant i, j \leqslant n\right\}$. Thus we can estimate (3.1) from below by

$$
\begin{aligned}
\inf \frac{1}{\sqrt{n}} \sum_{l=1}^{N}\left\{\left\|x_{l}\right\|_{2}+\left(\sum_{i=1}^{n}\right.\right. & \left.\left.\sum_{j=l_{l}+1}^{n} \frac{\left|x_{l}(i)\right|^{2}}{j}\right)^{1 / 2}\right\} \\
& \geqslant \frac{C}{\sqrt{n}} \inf \sum_{l=1}^{N}\left\{\left\|x_{i}\right\|_{2}+\left(\sum_{i=1}^{n}\left|x_{l}(i)\right|^{2} \ln \left(\frac{n}{l_{i}+1}\right)\right)^{1 / 2}\right\} \\
& \geqslant \frac{C}{\sqrt{n}} \inf \sum_{l=1}^{N}\left(\sum_{l=1}^{n}\left|x_{l}(i)\right|^{2}\left(1+\ln \left(\frac{n}{l_{i}+1}\right)\right)\right)^{1 / 2} .
\end{aligned}
$$

Since $\sum_{i=1}^{n} l_{i}=n$ and $l_{1} \geqslant l_{2} \geqslant \ldots \geqslant l_{n} \geqslant 0$, we have $l_{i} \leqslant n / i$. Thus the above is

$$
\geqslant \frac{C}{\sqrt{n}} \inf \sum_{l=1}^{N}\left(\sum_{i=1}^{n}\left|x_{l}(i)\right|^{2}(1+\ln i)\right)^{1 / 2}
$$

and this, by Hơlder's inequality and $\sum_{i=1}^{N}\left\|x_{l}\right\|_{2,1}=n$, is

$$
\geqslant \frac{C}{\sqrt{n}} \inf \sum_{l=1}^{N}\left(\sum_{i=1}^{n} \frac{\left|x_{l}(i)\right|}{\sqrt{i}}\right)\left(\sum_{i=1}^{n}|i(1+\ln i)|^{-1}\right)^{-1 / 2} \geqslant C^{\prime} \sqrt{\frac{n}{\ln \ln n}} .
$$


4. The positive projection constants of finite-dimensional Orlicz spaces. In this section $E$ will denote the expectation of random variables, and we will use the notations $x \vee y=\max \{x, y\}, x \wedge y=\min \{x, y\}$. Let $\left(\xi_{i}\right)_{i=1}^{n},\left(\eta_{i}\right)_{i=1}^{n}$ be two sequences of random variables on a probability space.

LemMa 4.1. Let $\left\{e_{i}\right\}$ be a 1-symmetric basis of an n-dimensional Banach space $F$. We have

(i) $\gamma_{\infty}^{+}(F) \leqslant n\left(E\left\|\sum_{i=1}^{n} \xi_{i} e_{i}\right\|_{F}\right)^{-1} \cdot \max _{\left\|x^{*}\right\|_{F^{*} \leqslant 1}} E \max _{1 \leqslant i \leqslant n}\left|\left\langle e_{i}, x^{*}\right\rangle \xi_{i}\right|$,

(ii) $\gamma_{\infty}^{+}(F) \geqslant n\left(E\left\|\sum_{i=1}^{n} \eta_{i} e_{i}^{*}\right\|_{F^{*}}\right)^{-1} \min _{\|x\|_{F} \geqslant 1} E \max _{1 \leqslant l \leqslant n}\left|\left\langle x, e_{i}^{*}\right\rangle \eta_{i}\right|$.

Proof. Inequality (i) is a continuous version of Lemma 2.5. To prove (ii) we use once again Lemma 2.5. For given $\varepsilon>0$ let $x_{1}, x_{2}, \ldots, x_{N}$ be vectors in $F$ such that $\sum_{l=1}^{N}\left\|x_{l}\right\|_{F}=n$ and

$$
\varepsilon+\gamma_{\infty}^{+}(F) \geqslant \max _{\left\|x^{*}\right\|_{F^{*}} \leqslant 1} \sum_{l=1}^{N} \max _{1 \leqslant i \leqslant n}\left|\left\langle e_{i}, x^{*}\right\rangle\left\langle x_{l}, e_{i}^{*}\right\rangle\right|
$$

Since the expectation of a random variable is not greater than its supremum, we have

$$
\begin{aligned}
& \max _{\left\|x^{*}\right\|_{F^{*}} \leqslant 1} \sum_{l=1}^{N} \max _{1 \leqslant i \leqslant n}\left|\left\langle e_{i}, x^{*}\right\rangle\left\langle x_{l}, e_{i}^{*}\right\rangle\right| \\
& \geqslant\left(E\left\|\sum_{i=1}^{n} \eta_{i} e_{i}^{*}\right\|_{F^{*}}\right)^{-1} E \sum_{l=1}^{n} \max _{1 \leqslant i \leqslant n}\left|\eta_{i}\left\langle x_{l}, e_{i}^{*}\right\rangle\right| \\
& \geqslant\left(E\left\|\sum_{i=1}^{n} \eta_{i} e_{i}^{*}\right\|_{F^{*}}\right)^{-1}:\left(\sum_{l=1}^{N}\left\|x_{i}\right\|_{F}\right) \min _{\|x\|_{F} \geqslant 1} E \max _{1 \leqslant i \leqslant n}\left|\left\langle x, e_{i}^{*}\right\rangle \eta_{i}\right| \\
&=n\left(E\left\|\sum_{i=1}^{n} \eta_{i} e_{i}^{*}\right\|_{F^{*}}\right)^{-1} \cdot \min _{\|x\|_{F} \geqslant 1} E \max _{1 \leqslant i \leqslant n}\left|\left\langle x, e_{i}^{*}\right\rangle \eta_{i}\right| .
\end{aligned}
$$

Combining this with the preceding inequality, we obtain the proof of (ii).

Let $M$ be an Orlicz function such that $\lim M(s) / s=0$ and let $\varrho$ $=M^{-1}(1)$. By $l_{n}^{M}$ we will denote the $n$-dimensional Orlicz space given by $M$, that is, $l_{n}^{M}$ coincides with $\boldsymbol{R}^{n}$ equipped with the norm whose unit ball is the set $\left\{\left(\alpha_{i}\right): \sum_{i=1}^{n} M\left(\left|\alpha_{i}\right|\right) \leqslant 1\right\}$.

With $M$ we associate a sequence $\left(\xi_{i}\right)_{i=1}^{n}$ of independent, nonnegative random variables such that the distribution of each $\xi_{i}$ satisfies $E \xi_{i} I_{y_{1}>t}$ $=t M(1 / t) \wedge 1 / \varrho$ for each $t>0$. Such a distribution exists and is unique. It has an atom at 0 and beyond this atom it has a density which is equal to $\left(s^{-2} M^{\prime}(1 / s)-s^{-1} M(1 / s)\right) I_{s \geq 0-1}$.
LemMA 4.2. For each sequence $\left(\alpha_{i}\right)$ in $l_{n}^{M}$ we have

$$
\left(1-e^{-1}\right)\left\|\left(\alpha_{i}\right)\right\|_{M} \leqslant E \max _{1 \leqslant i \leqslant n}\left|\alpha_{i}\right| \xi_{i} \leqslant 2\left\|\left(\alpha_{i}\right)\right\|_{M} .
$$

Proof. Since

$$
\max _{1 \leqslant i \leqslant n}\left|\alpha_{i}\right| \xi_{i} \leqslant 1+\sum_{i=1}^{n}\left|\alpha_{i}\right| \xi_{i} I_{\xi_{i}>\left|\alpha_{i}\right|^{-1}}
$$

we get

$$
E \max _{1 \leqslant i \leqslant n}\left|\alpha_{i}\right| \xi_{i} \leqslant 1+\sum_{i=1}^{n}\left|\alpha_{i}\right| E \xi_{i} I_{\xi_{i}>\left|\alpha_{i}\right|^{-1}} \leqslant 1+\sum_{i=1}^{n} M\left(\left|\alpha_{i}\right|\right) .
$$

Thus we deduce that if $\left\|\left(\alpha_{i}\right)\right\|_{M} \leqslant 1$ then $E \max \left|\alpha_{i}\right| \xi_{i} \leqslant 2$, which proves the right-hand inequality. On the other hand,

$$
\begin{aligned}
P\left(\max _{1 \leqslant i \leqslant n}\left|\alpha_{i}\right| \xi_{i} \geqslant s\right) & =1-\prod_{i=1}^{n}\left(1-P\left(\left|\alpha_{i}\right| \xi_{i} \geqslant s\right)\right) \\
& \geqslant 1-\exp \left(-\sum_{i=1}^{n} P\left(\left|\alpha_{i}\right| \xi_{i} \geqslant s\right)\right),
\end{aligned}
$$

for each $s \geqslant 0$. Since for each $x>0$ such that $1-e^{-1} \geqslant 1-e^{-x}$ we have $1-e^{-x} \geqslant\left(1-e^{-1}\right) x$, we deduce that if $P\left(\max _{1 \leqslant i \leqslant n}\left|\alpha_{i}\right| \xi_{i} \geqslant 1\right) \leqslant 1-e^{-1}$ then for all $s \geqslant 0$,

$$
P\left(\max _{1 \leqslant i \leqslant n}\left|\alpha_{i}\right| \xi_{i} \geqslant s\right) \geqslant\left(1-e^{-1}\right) \sum_{i=1}^{n} P\left(\left|\alpha_{i}\right| \xi_{i} \geqslant s \vee 1\right) .
$$

Since for each nonnegative random variable $\eta$ we have $E \eta I_{\eta>c}$ $=\int_{0}^{\infty} P(\eta>s \vee c) d s$, integrating the last inequality we get

$$
E \max _{1 \leqslant i \leqslant n}\left|\alpha_{i}\right| \xi_{i} \geqslant\left(1-e^{-1}\right) \sum_{i=1}^{n}\left|\alpha_{i}\right| E \xi_{i} I_{\xi_{i} \geqslant\left|\alpha_{i}\right|^{-1}}=\left(1-e^{-1}\right) \sum_{i=1}^{n} M\left(\left|\alpha_{i}\right|\right) \wedge\left|\alpha_{i}\right| / Q .
$$

Hence if $E \max _{1 \leqslant i \leqslant n}\left|\alpha_{i}\right| \xi_{i} \leqslant 1-e^{-1}$ then $P\left(\max _{1 \leqslant i \leqslant n}\left|\alpha_{i}\right| \xi_{i} \geqslant 1\right) \leqslant 1-e^{-1}$ and by what precedes $\sum_{i=1}^{n} M\left(\left|\alpha_{i}\right|\right) \wedge\left|\alpha_{i}\right| / \varrho \leqslant 1$. By the choice of $Q$ it follows that if $\left|\alpha_{i}\right| / Q \leqslant 1$ then $M\left(\left|\alpha_{i}\right|\right) \leqslant\left|\alpha_{i}\right| / Q$ and therefore $\sum_{i=1}^{n} M\left(\left|\alpha_{i}\right|\right) \wedge\left|\alpha_{i}\right| / Q \leqslant 1$ implies that $\sum_{i=1}^{n} M\left(\left|\alpha_{i}\right|\right) \leqslant 1$. This proves the left-hand inequality.

Let $M^{*}$ denote the complementary function of $M$ and let $\left(\xi_{i}^{*}\right), Q^{*}$ be defined for $M^{*}$ in the same way as $\left(\xi_{i}\right), \varrho$ are defined for $M$. We will assume 
in the sequel that the sequences $\left(\xi_{i}\right),\left(\xi_{i}^{*}\right)$ are independent. For two sequences $a_{n}, b_{n}$ of real numbers we will write

$$
a_{n} \sim b_{n} \text { up to a constant } C
$$

$$
C^{-1} a_{n} \leqslant b_{n} \leqslant C a_{n} \text { for all } n \text {. }
$$

Corollary 4.3. Up to a universal constant,

$$
\gamma_{\infty}^{*}\left(l_{n}^{M}\right) \sim \gamma_{\infty}^{+}\left(l_{n}^{M^{*}}\right) \sim n\left(E\left\|\left(\xi_{i}\right)\right\|_{M^{*}}\right)^{-1} \sim n\left(E\left\|\left(\xi_{i}^{*}\right)\right\|_{M}\right)^{-1} \sim n\left(E \max _{1 \leqslant i \leqslant n} \xi_{i} \xi_{i}^{*}\right)^{-1} .
$$

Proof. Since the norm of the canonical isomorphism between $l_{n}^{M^{*}}$ and $\left(l_{n}^{M}\right)^{*}$ does not exceed 2, we obtain by Lemmas 4.1 and 4.2

$$
\frac{1}{2}\left(1-e^{-1}\right) n\left(E\left\|\left(\xi_{i}\right)\right\|_{M^{*}}\right)^{-1} \leqslant \gamma_{\infty}^{+}\left(l_{n}^{M}\right) \leqslant 4 n\left(E\left\|\left(\xi_{i}^{*}\right)\right\|_{M}\right)^{-1} .
$$

Once again by Lemma 4.2 it follows that, up to the constant 2 ,

$$
E\left\|\left(\xi_{i}\right)\right\|_{M^{*}} \sim E \max _{1 \leqslant i \leqslant n} \xi_{i} \xi_{i}^{*} \sim E\left\|\left(\xi_{i}^{*}\right)\right\|_{M} .
$$

This proves Corollary 4.3.

To estimate the order of $E \max _{1 \leqslant i \leqslant n} \xi_{i} \xi_{i}^{*}$ let us introduce the function

$$
H(u)=u \underset{\substack{0<s<e^{2}, 0<t<e^{*} \\ s i<u}}{\int}(s t)^{-1}\left(M^{\prime}(s)-M(s) / s\right)\left(M^{* \prime}(t)-M^{*}(t) / t\right) d s d t
$$

and let $G=H^{-1}$ be its inverse function. Easy computations yield

$$
E \xi_{i} \xi_{i}^{*} I_{\xi_{i} \xi_{i}^{*}>u}=u H(1 / u) .
$$

Remark. If $M$ or $M^{*}$ satisfies the $\Delta_{2}$ condition then $H$ is equivalent in the sense of Orlicz functions to the function

$$
\bar{H}(u)=\int_{\substack{0<s<e, 0<t<e \\ s t<u}} M^{\prime}(s) M^{* \prime}(t) d s d t .
$$

Another formula for $H$ is the following one:

$$
H(u)=u \int_{\substack{0<s<0,0<<<Q^{*} \\ s t<u}}(s t)^{-2} M^{*}\left(M^{\prime}(s)\right) M\left(M^{* \prime}(t)\right) d s d t .
$$

By Lemma 4.2 we have for each sequence $\left(\alpha_{i}\right)$ of real numbers

$$
\frac{1}{2}\left\|\left(\alpha_{i}\right)\right\|_{H} \leqslant E \max _{1 \leqslant i \leqslant n}\left|\alpha_{i}\right| \xi_{i} \xi_{i}^{*} \leqslant 2\left\|\left(\alpha_{i}\right)\right\|_{H} .
$$

Putting in the above inequalities $\alpha_{i}=1, i=1,2, \ldots, n$, and applying Corollary 4.3, we obtain the following estimate:

COROLlarY 4.4. Up to a universal constant,

$$
\gamma_{\infty}^{+}\left(l_{n}^{M}\right) \sim n G(1 / n)
$$

The order of $\gamma_{\infty o}^{+}\left(l_{n}^{p}\right)$ was computed in [7]. Here we want to give more precise estimates. For the case of $l_{n}^{p}$ it is better to consider slightly different random variables from those we introduce in the general case. For a fixed $p$ let $\left(\xi_{i}\right)_{i=1}^{n}$ be a sequence of independent random variables each of them distributed by

$$
P\left(\xi_{i} \leqslant t\right)=\exp \left(-c_{p} t^{-p}\right) \text { for } t>0, \quad \text { where } c_{p}=\Gamma((p-1) / p)^{-p},
$$

and let $\left(\xi_{i}^{*}\right)_{i=1}^{n}$ be such a sequence for $p^{*}=p /(p-1)$. If $\sum_{i=1}^{n}\left|\alpha_{i}\right|^{p}=1$ then the random variable $\max _{1 \leqslant i \leqslant n}\left|\alpha_{i}\right| \xi_{i}$ is equally distributed with $\xi_{1}$, and therefore we have

$$
E \max _{1 \leqslant i \leqslant n}\left|\alpha_{i}\right| \xi_{i}=E \xi_{1}=1 .
$$

By Lemma 4.1 this gives the following inequalities:

$$
n\left(E\left\|\left(\xi_{i}\right)\right\|_{p^{*}}\right)^{-1} \leqslant \gamma_{\infty}^{+}\left(l_{n}^{p}\right) \leqslant n\left(E\left\|\left(\xi_{i}^{*}\right)\right\|_{p}\right)^{-1} .
$$

This implies that $E\left\|\left(\xi_{i}^{*}\right)\right\|_{p} \leqslant E\left\|\left(\xi_{i}\right)\right\|_{p^{*}}$ and by symmetry the last two expressions are equal. As a consequence of this we immediately obtain the following result:

Corollary 4.5. For $1<p<\infty$ and all $n \in N$ we have

$$
\gamma_{\infty}^{+}\left(l_{n}^{p}\right)=\gamma_{\infty}^{+}\left(l_{n}^{p^{*}}\right)=n\left(E\left\|\left(\xi_{i}^{*}\right)\right\|_{p}\right)^{-1}=n\left(E\left\|\left(\xi_{i}\right)\right\|_{p^{*}}\right)^{-1}=n\left(E \max _{1 \leqslant i \leqslant n} \xi_{i} \xi_{i}^{*}\right)^{-1} .
$$

Thus to compute $\gamma_{\infty}^{+}\left(l_{n}^{p}\right)$ we may assume that $p<p^{*}$, or $p=2$. If $p<p^{*}$ then $\xi^{* p}$ has finite moments of order greater than 1 and hence by the Law of Large Numbers we have

$$
\lim _{n \rightarrow \infty} n^{-1} \sum_{i=1}^{n} \xi_{i}^{* p}=E \xi_{1}^{* p}=\Gamma\left(1 / p^{*}\right)^{-p} \Gamma(2-p),
$$

the convergence being in $L_{1}$. This shows that

$$
\lim _{n \rightarrow \infty} n^{-1 / p} E\left\|\left(\xi_{i}^{*}\right)\right\|_{p}=\lim _{n \rightarrow \infty} E\left(n^{-1} \sum_{i=1}^{n} \xi_{l}^{* p}\right)^{1 / p}=\Gamma\left(1 / p^{*}\right)^{-1} \Gamma(2-p)^{1 / p} .
$$

If $p=2$ then by arguments as in Theorem 2, Ch. VII, $\S 7$ of [3] we obtain

$$
\lim _{n \rightarrow \infty} E\left(\frac{\sum_{i=1}^{n} \xi_{i}^{* 2}}{n \ln n}\right)^{1 / 2}=\frac{1}{\sqrt{\pi}} \text {. }
$$


Corollary 4.6.
(i) If $p=2$ then $\lim _{n \rightarrow \infty} \gamma_{\infty}^{+}\left(l_{n}^{2}\right)\left(\frac{n}{\ln n}\right)^{-1 / 2}=\sqrt{\pi}$.
(ii) If $p<p^{*}$ then $\lim _{n \rightarrow \infty} \gamma_{\infty}^{+}\left(l_{n}^{p}\right) n^{1 / p-1}=\Gamma\left(1 / p^{*}\right) \Gamma(2-p)^{-1 / p}$.

\section{References}

[1] D. Dacunha-Castelle, Variables aléatoires échangeables et espaces d'Orlicz, Séminaire Maurey-Schwartz, 1974-1975, École Polytechnique, exposés no. 10 et 11.

[2] W. Feller, An Introduction to Probability Theory and Its Applications, Vol. I, John Wiley \& Sons, New York 1966.

[3] W. Feller, An Introduction to Probability Theory and Its Applications, Vol. II, John Wiley \& Sons, New York 1966.

[4] E. D. Gluskin, Norm estimates of certain p-absolutely summing operators, Funktsional. Anal. i Prilozhen. 12 (2) (1978), 24-31.

[5] J. Lindenstrauss and L. Tzafriri, Classical Banach Spaces, I, II, Springer-Verlag, 1977 and 1979.

[6] C. Schutt, On the Banach-Mazur distance of finite-dimensional symmetric Banach spaces and the hypergeometric distribution, Studia Math. 72 (1982), 109-129.

[7] C. Schutt, On the positive projection constant, Studia Math. 78 (1984), 185-198.

INSTYTUT MATEMATYKI UNIWERSYTETU WARSZAWSKIEGO

INSTITUTE OF MATHEMATICS, WARSAW UNIVERSITY

PKiN IX p., Warsaw, Poland

and

CHRISTIAN-ALBRECHTS-UNIVERSITÄT ZU KIEL

MATHEMATISCHES SEMINAR

Olshausenstraße 40-60, D 2300 Kiel 1, West Germany

Received February 2, 1984

Revised version June 30, 1984 